\title{
MR Imaging of Cerebral Extraventricular Neurocytoma: A Report of 9 Cases
}

\author{
K. Liu, G. Wen, X.-F. Lv, Yan-Jia Deng, Yong-Jian Deng, G.-Q. Hou, X.-L. Zhang, L.-J. Han, and J.-L. Ding
}

\begin{abstract}
SUMMARY: Extraventricular neurocytoma is a rare entity, most frequently occurring in brain parenchyma outside the ventricular system. The purpose of this study was to characterize the MR imaging findings in a series of 9 patients with EVN verified by results of pathologic examination. All 9 EVNs were solitary and intracranially located. Eight lesions were well demarcated, and 3 showed intratumoral hemorrhage. The solid parts of 7 tumors were primarily isointense on T1-weighted images and heterogeneously enhanced on TIWI with contrast. Although cerebral EVNs can present a wide spectrum of appearances on MR, the imaging patterns appear to vary according to anatomic location and cellularity. Lesions in frontal or parietal lobes often present as well-demarcated large masses with cystic degeneration, hemorrhage, mild-to-moderate edema, and inhomogeneous enhancement. Moreover, the general isointensity of the solid parts of EVN on TTWI may be of some specificity.
\end{abstract}

ABBREVIATIONS: $\mathrm{CN}=$ central neurocytoma; $\mathrm{EVN}=$ extraventricular neurocytoma; $\mathrm{WHO}=$ World Health Organization

$\mathbf{C}^{\wedge}$ Ns were first described by Hassoun et al in $1982^{1}$ and are uncommon benign tumors of the CNS. Such tumors typically arise within the lateral ventricles of young adults. Extraventricular CN has been termed "EVN." Although this entity shows biologic behavior and histopathologic characteristics similar to those of CNs, it was proposed as an International Classification of Diseases-O code (9506/1), according to 2007 WHO classification, ${ }^{2}$ identical to that of $\mathrm{CN}$.

The defining histologic feature of EVN is its composition of monotonous neoplastic cells with round, regular nuclei embedded in the neuropil, ${ }^{2-4}$ which usually resembles oligodendrogli-

Received May 5, 2012; accepted after revision June 12.

From the Medical Imaging Center (K.L., G.W., X.-L.Z.) and Department of Pathology (Yong-Jian D.), Nanfang Hospital, Southern Medical University, Guangzhou, P.R. China; Department of Medical Imaging and Interventional Radiology (X.-F.L., L.-J.H.), Cancer Center, Sun Yat-Sen University, Guangzhou, P.R. China; Department of Radiology (Yan-Jia D.), The 5th Affiliated Hospital of Sun Yat-Sen University, Zhuhai, P.R. China; Department of Medical Image Center (G.-Q.H.), Nanshan Hospital, Shenzhen, P.R. China; Department of Radiology (X.-L.Z.), Dongguan Kanghua Hospital, Dongguan, P.R. China; and Department of Radiology (J.-L.D.), The People's Hospital of Futian Shenzhen, Shenzhen, P.R. China.

K. Liu and G. Wen contributed equally to this work.

Please address correspondence to Xue-Lin Zhang, MD, Department of Medical Imaging Center, Nanfang Hospital, Southern Medical University, No. 1838, Guangzhou Ave. North, Guangzhou 510515, P.R. China, and Department of Radiology, Dongguan Kanghua Hospital, Donggua 523000, P.R. China; e-mail: zhangxuelin 2010@163.com; or Lu-Jun Han, MD, Department of Medical Imaging and Interventional Radiology, Cancer Center, Sun Yat-Sen University, No.651 Dong Feng Dong Rd, Guangzhou 510060, P.R. China; e-mail: hanlujun2000@126.com.

- Indicates open access to non-subscribers at www.ajnr.org

http://dx.doi.org/10.3174/ajnr.A3264 oma and other tumors. ${ }^{3,5,6}$ The diagnosis of EVN can be aided by imaging features, such as a well-defined tumor margin and "dotted" calcifications on CT scan. ${ }^{7}$

To date, on the basis of clinical or histopathologic features, approximately 70 cases of this entity have been reviewed. ${ }^{4-6,8-13}$ Only a few radiologic studies emphasizing the $\mathrm{CT}$ and MR imaging findings of EVN are available, however, ${ }^{7,14-20}$ and only in scattered case reports and small series. We report 9 cases of cerebral EVNs, the largest collection of EVNs among the radiologic studies of this entity in the literature to date.

\section{MATERIALS AND METHODS}

Our study was approved by our institutional review boards. We retrospectively reviewed MR and CT images, results of pathologic examinations, and clinical data of 9 patients ( 4 women and 5 men; 16-59 years old; median age, 40 years) diagnosed with EVN at our institution from 2005 to 2011. The diagnosis of EVN was established on the basis of the combination of histologic and immunohistochemical evidence of neurocytoma and an extraventricular location proved at surgery.

All 9 patients underwent pretreatment MR examination, and 3 underwent pretreatment CT examinations. MR imaging was performed on 7 patients by using a 1.5T scanner (Magnetom VP; Siemens, Erlangen, Germany), and 2 patients were studied by using a 3T scanner (Signa Excite, GE Healthcare, Milwaukee, Wisconsin). Pregadolinium T1WI (TR, 450-600 ms; TE, 12-16 ms), T2WI (TR, 4500-5500 ms; TE, 100-20 ms), and gadoliniumenhanced $(0.1-0.2 \mathrm{mmol} / \mathrm{kg}) \mathrm{T} 1 \mathrm{WI}$ were acquired. Noncontrast 

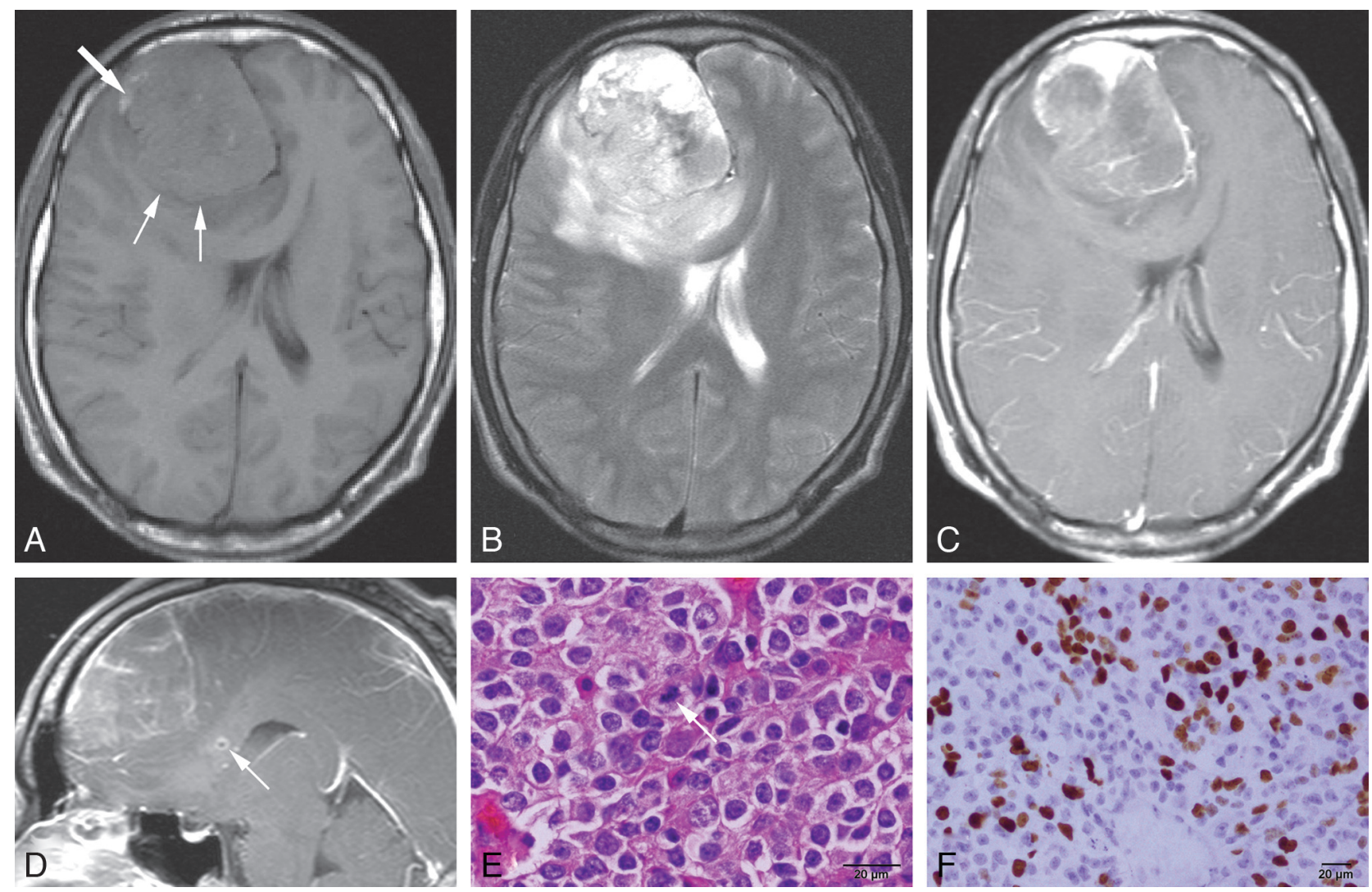

FIG 1. MR images of a 33-year-old man with atypical EVN of the right frontal lobe. A, Axial precontrast TIWI shows a well-demarcated large mass in the right frontal lobe (small arrows), isointense to gray matter dotted with small foci of hyperintensity representing hemorrhage (large arrow). B, Axial T2WI reveals mixed hyperintense to hypointense components with moderate peritumoral edema. $C$ and $D$, Axial and sagittal postcontrast TIWI demonstrate a mass with uneven and patchy enhancement. A small focus of infiltration adjacent to the mass is noted in corpus callosum (arrow, D). E, Hematoxylin and eosin staining shows the composition of monotonous small tumor cells with regular round nuclei, and mitosis (arrow). F, Results of immunohistochemical assays demonstrate Ki-67 > 30\%.

$(n=3)$ and contrast $(n=2)$ CT images were obtained by using a section thickness of $3 \mathrm{~mm}, 120 \mathrm{kV}$, and 150-200 mAs before and after the intravenous administration of contrast agent $(300 \mathrm{mg}$ I/mL; 1.5-2.0 mL/kg; Omnipaque, GE Healthcare).

All the MR and CT images were retrospectively reviewed by 2 neuroradiologists blinded to histologic diagnosis, and decisions were reached by consensus. Specific imaging features included location, size, internal architecture, margin, focal infiltration, perilesional edema, attenuation, signal intensity, and enhancement of the tumors. The degree of perilesional edema/enhancement of the solid part of each tumor was classified as none, mild, moderate, or extensive/strong.

\section{RESULTS}

\section{Clinical Data}

Patient symptoms included headache $(n=5)$, dizziness $(n=3)$, visual abnormality $(n=3)$, seizures $(n=1)$, vomiting $(n=1)$ and hypodynamia of limbs $(n=1)$. Duration of symptoms ranged from 2 weeks to 16 years at diagnosis. Total resection was performed in 7 patients, and 1 received subtotal resection. The surgical history of 1 patient was unavailable. To date, except for 2 patients lost to follow-up, all remaining patients are alive. The 1 patient who underwent subtotal tumor resection was alive with remnant tumor, and recurrence was confirmed by CT and MR examination in 1 patient. No evidence of recurrence was observed in the other 5 patients.

\section{Imaging Findings}

All lesions were solitary. Two were located in the frontal lobe (Fig 1 ), and 1 each was found in the parietal lobe (Fig 2), temporal lobe (Fig 3), thalamus, hypothalamus, tectum of midbrain, pineal, and sellar/suprasellar region (Fig 4).

Tumors ranged from 1.8 to $8.6 \mathrm{~cm}$ in greatest diameter, with a mean diameter of $4.7 \mathrm{~cm}$. The mean diameter of the frontal and parietal lesions was $7.2 \mathrm{~cm}$. Seven of 9 lesions were well-demarcated solid ( $n=2)$ or complex $(n=5)$ masses of either ovoid ( $n=$ $3)$ or irregular $(n=4)$ shape. One tumor was small, ovoid, and purely cystic. One tumor presented solely as swelling of the tectum of the midbrain with no distinct margin. Focal infiltrations were noted in the cavernous sinus and optic chiasma adjacent to the sellar lesion (Fig 4C), and in the corpus callosum adjacent to the frontal tumor (Fig 1D).

On T1WI, 3 lesions showed complex hyperintense areas representing hemorrhage (Figs $1 A$ and $2 A$ ), and 5 showed hypointense cystic degeneration. The solid parts (including the cyst wall of the purely cystic case) of 7 lesions were predominantly isointense with small hyperintense and/or hypointense foci compared 

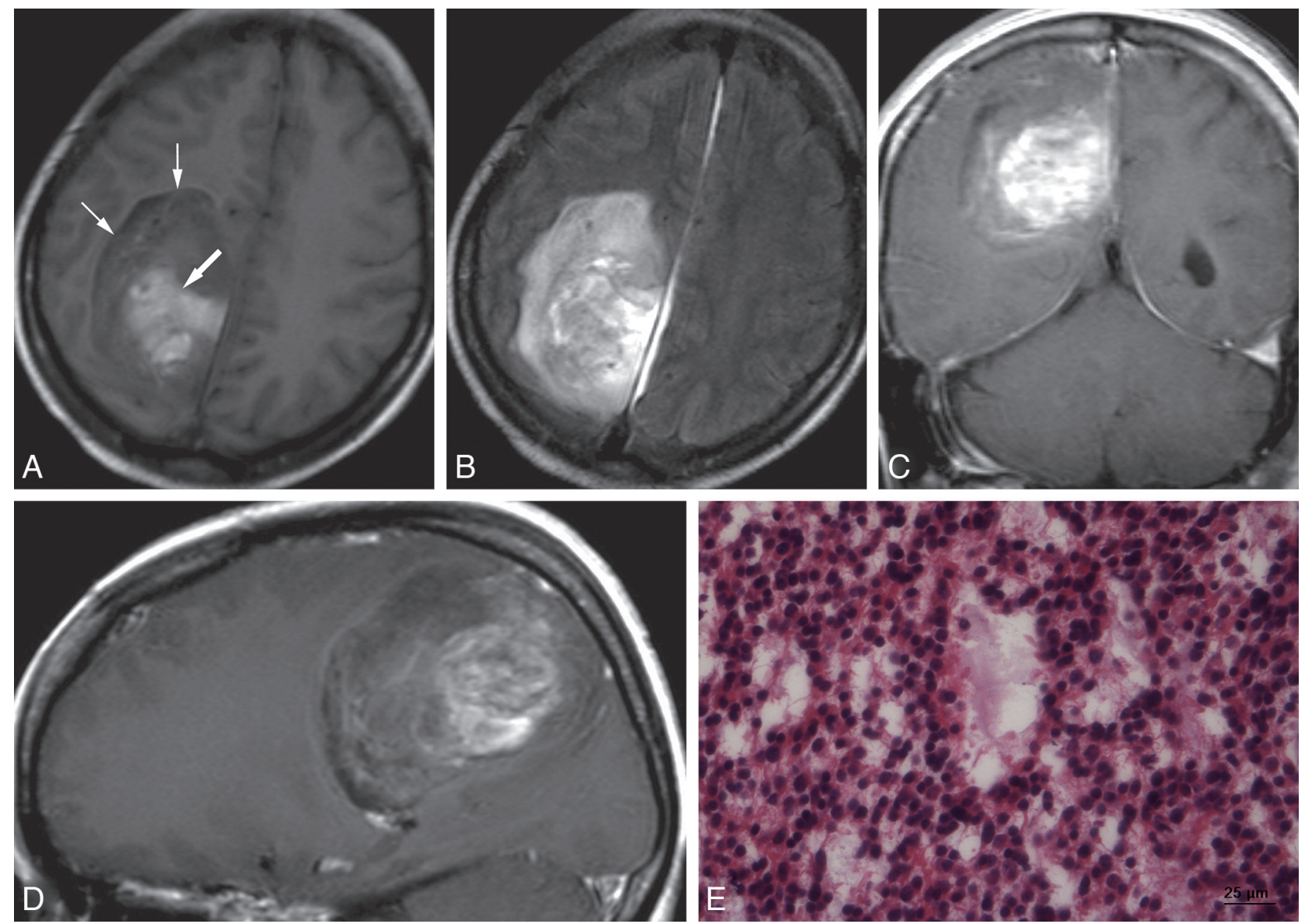

FIG 2. MR images of a 43-year-old woman with EVN of the right parietal lobe. A, Axial TIWI shows a hypointense well-demarcated large mass (small arrows) with hyperintensity of its central area due to hemorrhage (large arrow). B, Axial FLAIR image shows a heterogeneous hyperintense mass with only slight peritumoral edema. $C$ and $D$, Coronal and sagittal postcontrast T1WI show mild inhomogeneous "zebra-like" enhancement of the solid portion. $E$, Hematoxylin and eosin staining shows extensive microcystic change.

with gray matter (Figs $1 A$ and $4 A$ ). Two showed hypointensity of the solid parts (Figs $2 A$ and $3 A$ ). In comparison, the signal intensity was more heterogeneous and predominantly hyperintense on T2WI and FLAIR images in all 9 lesions (Figs 1, 2, and 4). Perilesional edema was noted in 4 patients ( 3 moderate and 1 mild).

Contrast-enhanced T1WI demonstrated heterogeneously mild ( $n=2$, Fig $2 C,-D)$, moderate $(n=3$, Fig $1 C, D)$, or strong ( $n=2$, Fig $4 C)$ tumoral enhancement. The enhancement patterns included "rim and ringlike," "ringlike with mural nodular," "patchy" (Fig 1C, -D), and "zebra" (Fig 2D). No obvious enhancement, compared with adjacent brain tissue, was noted in 2 cases (Fig 3C, -D). Calcification was confirmed on CT in 2 of 3 patients studied, and the solid parts of the tumors showed intermediate $(n=2)$ or slightly increased attenuation $(n=1$, Fig $4 D)$.

\section{Pathology Findings}

All 9 EVN specimens were composed of monotonous tumor cells containing regular, round nuclei, as revealed by light microscopy (Figs $1 E$ and $2 E$ ). Positive antibody reactions to synaptophysin, glial fibrillary acidic protein, chromogranin A, neuronal nuclear antigen, and neurofilament were $100 \%, 66.7 \%, 57.1 \%, 66.7 \%$, and $57.1 \%$, respectively. One case was identified as atypical EVN with a labeling index for Ki-67 greater than 30\% (Fig $1 F$ ).

\section{DISCUSSION}

The first EVN was reported by Ferreol et al in $1989^{10}$ as an extremely rare neoplasm. This entity, corresponding to WHO Grade II tumor, has gained increased recognition over the past 2 decades. Although no exact incidence of EVN has been reported, upon review of approximately 7000 patients with intracranial tumors admitted to our institutions within the same period as our series, we estimated the incidence of cerebral EVN to be $0.13 \%$. EVN occurs in patients between 2 and 76 years of age (median age, 34 years), and children and young adults are more frequently affected. EVNs show no obvious sex predilection. ${ }^{4}$ Our series of 4 women and 5 men, ranging in age from 16 to 59 years (mean, 36.9 years), is consistent with the literature.

The clinical presentation of cerebral EVN is usually nonspecific, depending primarily on the location of the tumor and whether it exerts a mass effect. Patients usually present with headache, seizures, diplopia, and vomiting. ${ }^{4,9}$ Visual impairment (which was present in our case of sellar/suprasellar EVN) occurred in only 2 other cases reported in the literature. ${ }^{7,8}$ The visual impairment was secondary to the invasion of optic nerve and/or optic chiasma by tumor.

Although EVN has been reported in almost every extraventricular CNS site, the most common tumoral location is the 

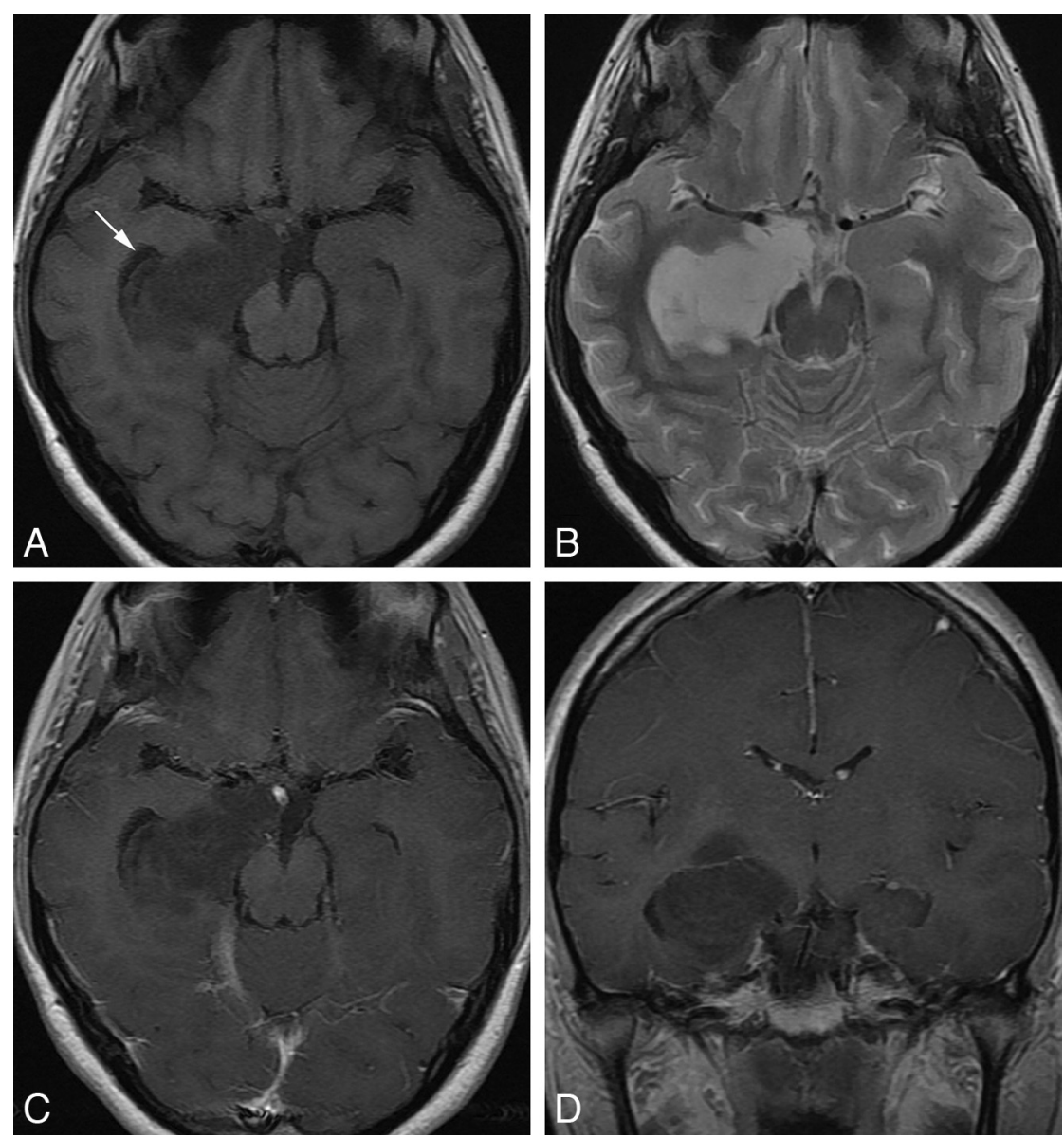

FIG 3. MR images of a 20-year-old woman with EVN of the right temporal lobe. A, Axial TIWI shows a homogeneous hypointense lesion located deep in the right temporal lobe. The temporal horn of right lateral ventricle is displaced (arrow). B, Axial T2WI image shows markedly hyperintense signal within the lesion approaching that of the CSF. No obvious perilesional edema is observed. $C$ and $D$, Axial and coronal postcontrast TIWI show no obvious enhancement.

frontal lobe, followed by the parietal, temporal, and occipital lobes. ${ }^{4,9,15}$ Unusual intracranial locations include the thalamus and hypothalamus, ${ }^{4,9}$ pons, ${ }^{21}$ sellar region, ${ }^{7,8}$ pineal gland, ${ }^{17}$ and base of the skull. ${ }^{22}$ The location diversity of EVN mentioned in other studies ${ }^{3,8,22}$ is also confirmed by our series.
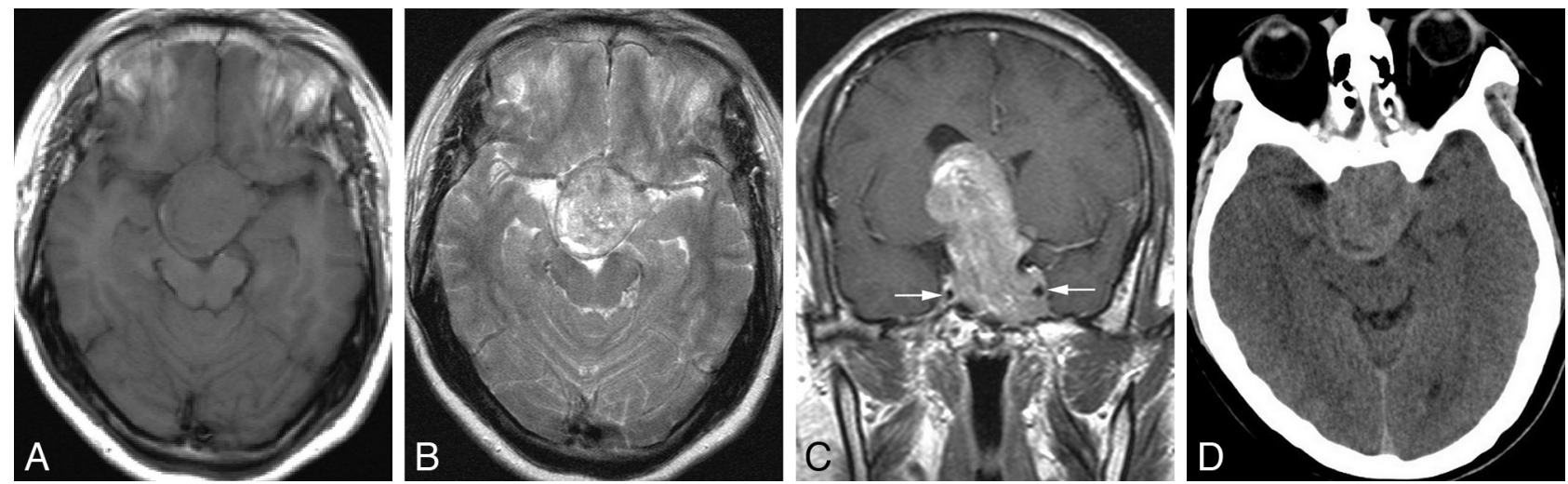

FIG 4. MR and CT images of a 40-year-old man with EVN of the sellar and suprasellar region. $A$, Axial TTWI shows a lesion with primarily isointense signal. $B$, Axial T2WI shows a mixed isointense to hyperintense lesion. C, Coronal postcontrast TIWI demonstrates the heterogeneously strong enhancement of the lesion and the transverse infiltration toward vascular structures on both sides (arrows). D, Axial precontrast CT image shows a lesion with slightly increased attenuation and small foci of decreased attenuation of cystic degeneration.
The distinct immunohistochemical feature of EVN is strong and diffuse synaptophysin immunoreactivity, ${ }^{4}$ which is helpful in confirming the diagnosis. Histologically atypical EVNs exhibit a MIB-1 labeling index of $>2 \%$ or atypical histologic features. ${ }^{4,11}$ Such EVNs are extremely rare, and fewer than 20 cases have been reported. ${ }^{12}$

Available studies on the MR imaging features of EVN are scarce. We found and reviewed a total of 55 cases of intracranial EVNs (cerebellar cases were not included) with specific radiologic descriptions in neuroimaging literature to date. According to previous findings, EVN frequently presents as a well-demarcated $(25 / 33,75.8 \%)$ mass of the frontal $(20 / 55,36.4 \%)$ or parietal lobe $(11 / 55,20 \%)$, with cystic degeneration (32/ $55,58.2 \%)$, perilesional edema (17/33, $51.5 \%)$, calcification $(13 / 38,34.2 \%)$, and hemorrhage $(5 / 24,20.8 \%)$. The tumor usually shows nonspecific signal intensity, varous degrees and patterns of enhancement lar to that of low-grade pilocytic astrocytoma. ${ }^{4,14,16}$

Most of these findings parallel our series, because a well-defined margin was seen in 8 of our $9(88.9 \%)$ cases. Six $(66.7 \%)$ showed cystic degeneration, 3 revealed hemorrhage $(33.3 \%)$, and 4 (44.4\%) showed perilesional edema. Mild to strong enhancement was noted in $7(77.8 \%)$ cases. In addition, new findings in our series concerned focal infiltration and complex internal architecture, isointensity of the solid part on T1WI, and nonenhancement of some tumors. , $92.5 \% ; 3[7.5 \%]$ showed no enasly enhanced), and variable appear- 
Although the margin in most of our tumors of cerebral parenchyma was generally well defined, focal infiltration (scarcely mentioned in previous reports ${ }^{4,23}$ ) occurred in the corpus callosum adjacent to 1 lesion, which also exhibited Ki-67 > 30\%, histologically identified as an atypical EVN. This finding suggests that atypia or high mitotic index, previously recognized as a poor prognostic sign, ${ }^{6,11,13}$ may also be responsible for aggressive characteristics such as an infiltrative pattern on MR images. This infiltration observed on MR may predict recurrence after surgery. Another case demonstrated complex intratumoral architecture, inhomogeneous rim-like and ring-like enhancement, diffuse intratumoral hemorrhage, and moderate perilesional edema. These findings parallel 2 cases in Yang et al's series, ${ }^{7}$ supporting the diagnosis of a high-grade lesion such as glioblastoma on MR imaging. Therefore, prior reports concluding that EVN radiographically has a low-grade appearance may not apply to all cases.

General isointensity or slight hyperintensity on T1WI of the solid part of EVN (7/9 cases, $77.8 \%$; including the cyst wall of the pineal case), is distinct from the hypointensity found in glioma. Along with intermediate or slightly increased CT attenuation, these signs are usually found in $\mathrm{CN}^{24}$ (100\% in the 8 cases in Wichmann et al's study ${ }^{25}$ ) and may correlate with the compact regularity of tumor cell arrangement seen in both EVN and CN. This hypothesis may be further supported by the increased signal intensity of these 2 tumor types on DWI, ${ }^{7,26}$ for attenuated cellularity of tumor tissue may lead to restricted diffusion according to previous studies. ${ }^{27,28}$ By contrast, our 1 case of solid tumor that was hypointense on T1WI showed extensive microcystic change by light microscopy, suggesting that the MR signal intensity and CT attenuation of EVNs are dependent upon their cellularity.

Total lack of enhancement was observed in 2 of our cases (28.6\%) with surgically proved poor blood supplies. This confirms previous findings of nonenhancing hypovascular extraventricular neurocytoma. ${ }^{15,29}$

Although EVN appears to possess a wide spectrum of imaging patterns, especially with regards to size and shape, lesions located in either the frontal or parietal lobes tend to show radiographic consistency, both in our series and in previous reports: a welldemarcated, large $(7.2 \pm 1.5 \mathrm{~cm})$ mass with cystic degeneration, mild-to-moderate edema, intratumoral hemorrhage, and heterogeneous enhancement. In addition, EVN of the sellar/suprasellar region in our series exhibited focal infiltration of the cavernous sinus and optic chiasma but remained "nonaggressive" on results of histologic examination. Both previous cases in the literature of sellar EVNs also showed focal cavernous sinus invasion but remained nonaggressive on results of histologic examination. ${ }^{7,8}$ Therefore, this invasive growth pattern, which contradicts that of EVNs of cerebral parenchyma, may be a feature of sellar lesions. Unfortunately, neither of the previous studies mentioned a possible underlying mechanism. To our knowledge, pilocytic astrocytoma, a typically well-circumscribed cerebral tumor when it arises within the optic chiasm, can exhibit similar peripheral infiltrative extension but remain nonaggressive on results of histologic examination because of the lack of clear demarcation between tumor and normal tissue. ${ }^{30,31}$ Perhaps the same locational feature, ie, the lack of demarcation (which usually confines tumor growth and helps to form a well-defined margin) may be respon- sible for the infiltrative growth pattern of EVN in the sellar and suprasellar regions. Moreover, our pineal EVN presented as a small mass with purely cystic architecture, which mimics the only other previous case of pineal EVN ever reported. ${ }^{17}$ These findings suggest that the imaging patterns of EVN may vary according to anatomic location.

The differential diagnosis of EVN should include oligodendroglioma, astrocytoma, dysembryoplastic neuroepithelial tumor, pituitary adenoma, and congenital pineal cyst. From a radiologic perspective, EVN mimics both low- and high-grade astrocytoma and oligodendroglioma because of its shape and high incidence of cystic degeneration and calcification. Be that as it may, compared with EVN, oligodendrogliomas and astrocytomas usually lack a well-defined margin, ${ }^{7}$ and the solid parts of these tumors are primarily hypointense on T1WI and demonstrate decreased attenuation on CT.

EVN sometimes shows neither enhancement nor perilesional edema, especially in the temporal lobe, and thus mimics dysembryoplastic neuroepithelial tumor on imaging. ${ }^{15,29}$ The characteristic cortical location of dysembryoplastic neuroepithelial tumor, however, may be of differential significance. EVN of the sellar/suprasellar region should be differentiated from pituitary adenoma, because the latter seldom shows increased CT attenuation and heterogeneous enhancement on contrast-enhanced images. In addition, congenital pineal cyst should also be included in the differential diagnosis because EVN may present as a pure cyst in the pineal region. The intracystic hemorrhage and hyperintense signal of the intracystic fluid on FLAIR may suggest the diagnosis of EVN. ${ }^{17}$

Limitations of our study lie in the lack of the diffusionweighted and spectroscopy data of EVN. Two cases of EVN with diffusion-weighted imaging have been reported; one indicated increased signal intensity of solid tumor. ${ }^{7}$ This may be related to the compact regularity of tumor cell arrangement in that increased cellularity is considered to be responsible for restricted diffusion of tumors in a number of studies. ${ }^{27,28}$ By contrast, the other case, with no diffusion restriction, showed much looser cell arrangement by light microscopy, which is different from the conventional histologic feature of EVN. ${ }^{29}$ Thus, ADC and DWI of EVN may consequently be of some differential diagnostic value, because restricted diffusion is less obvious in some tumors (eg, pilocytic astrocytoma, ${ }^{28}$ which mimics some EVNs on conventional MR images).

To date, there are $3 \mathrm{MR}$ spectroscopy studies of EVN, and strongly decreased or no NAA peak and prominent choline peak were shown. ${ }^{18-20}$ Presence of NAA, which has been noted as a possible characteristic feature of $\mathrm{CN}$ both in vivo and in vitro, ${ }^{32}$ is not prominent in EVN, possibly because even the highly differentiated cells of EVN may be too immature to produce NAA. ${ }^{19}$ These findings may further suggest that EVN is a different type of tumor from CN. ${ }^{18}$

\section{CONCLUSIONS}

Cerebral EVN can occur in diverse intracranial sites. It appears to possess a wide spectrum of imaging patterns on MR and CT, which may vary according to cellularity and anatomic locations (especially in the pineal and sellar/suprasellar regions). EVN le- 
sions of the frontal or parietal lobes tend to show radiographic consistency (ie, a well-demarcated, large mass with various degrees of cystic degeneration, mild-to-moderate perilesional edema, frequent intratumoral hemorrhage, and inhomogeneous enhancement). Moreover, the general isointensity on T1WI and intermediate or slightly increased CT attenuation exhibited by the solid parts of these tumors may be of some differential significance.

\section{ACKNOWLEDGMENTS}

We thank Min Song, of the Southern Medical University Library, Zhibin Li, of the 302 Military Hospital of China, and Xiao Liu, of the Peking University Third Hospital, for their technical assistance.

Disclosures: Kai Liu, Ge Wen, Yong-Jian Deng, Xue-Lin Zhang—RELATED: Grant: Medical Research Foundation of Guangdong Health Commission of Guangdong Province (code: A2009406); Technology Program of Guangzhou Baiyun of China (code: 2011-KZ-92).

\section{REFERENCES}

1. Hassoun J, Gambarelli D, Grisoli F, et al. Central neurocytoma. An electron-microscopic study of two cases. Acta Neuropathol 1982;56:151-56

2. Louis DN, Ohgaki H, Wiestler OD, et al. The 2007 WHO classification of tumours of the central nervous system. Acta Neuropathol 2007;114:97-109

3. Sharma MC, Deb P, Sharma S, et al. Neurocytoma: a comprehensive review. Neurosurg Rev 2006;29:270-85

4. Brat DJ, Scheithauer BW, Eberhart CG, et al. Extraventricular neurocytomas: pathologic features and clinical outcome. Am J Surg Pathol 2001;25:1252-60

5. Mut M, Güler-Tezel G, Lopes MB, et al. Challenging diagnosis: oligodendroglioma versus extraventricular neurocytoma. Clin Neuropathol 2005;24:225-29

6. Sakurada K, Akasaka M, Kuchiki H, et al. A rare case of extraventricular neurocytoma. Brain Tumor Pathol 2007;24:19-23

7. Yang GF, Wu SY, Zhang LJ, et al. Imaging findings of extraventricular neurocytoma: report of 3 cases and review of the literature. AJNR Am J Neuroradiol 2009;30:581-85

8. Wang YY, Kearney T, du Plessis D, et al. Extraventricular neurocytoma of the sellar region. Br J Neurosurg 2011;26:420-22

9. Giangaspero F, Cenacchi G, Losi L, et al. Extraventricular neoplasms with neurocytoma features. A clinicopathological study of 11 cases. Am J Surg Pathol 1997;21:206-12

10. Ferreol E, Sawaya R, de Courten-Myers GM. Primary cerebral neuroblastoma (neurocytoma) in adults. J Neurooncol 1989;7:121-28

11. Söylemezoglu F, Scheithauer BW, Esteve J, et al. Atypical central neurocytoma. J Neuropathol Exp Neurol 1997;56:551-56

12. Ghosal N, Dadlani R, Somorendra SS, et al. Atypical extraventricular neurocytoma: a rare and challenging case diagnosed on intraoperative cytology. Cytopathology 2012;23:270-73

13. Kane AJ, Sughrue ME, Rutkowski MJ, et al. Atypia predicting prog- nosis for intracranial extraventricular neurocytomas. J Neurosurg 2012;116:349-54

14. Amemiya S, Shibahara J, Aoki S, et al. Recently established entities of central nervous system tumors: review of radiological findings. J Comput Assist Tomogr 2008;32:279-85

15. Tortori-Donati P, Fondelli MP, Rossi A, et al. Extraventricular neurocytoma with ganglionic differentiation associated with complex partial seizures. AJNR Am J Neuroradiol 1999;20:724-27

16. Shin JH, Lee HK, Lee JK, et al. MR imaging and histopathologic findings of a case of cerebral ganglioneurocytoma. Korean J Radiol 2002;3:214-17

17. Ng P, Soo YS, Chaseling R, et al. Intraventricular neurocytoma. Australas Radiol 1996;40:125-33

18. Ueda F, Suzuki M, Matsui O, et al. Automated MR spectroscopy of intra- and extraventricular neurocytomas. Magn Reson Med Sci 2007;6:75-81

19. Möller-Hartmann W, Krings T, Brunn A, et al. Proton magnetic resonance spectroscopy of neurocytoma outside the ventricular region-case report and review of the literature. Neuroradiology 2002;44:230-34

20. Kanamori M, Kumabe T, Shimizu H, et al. ${ }^{201}$ Tl-SPECT, ${ }^{1}$ H-MRS, and MIB-1 labeling index of central neurocytomas: three case reports. Acta Neurochir (Wien) 2002;144:157-63

21. Soontornniyomkij V, Schelper RI. Pontine neurocytoma. J Clin Pathol 1996;49:764-65

22. Shidoh S, Yoshida K, Saitoh K, et al. Extraaxial neurocytoma in the skull base. Brain Tumor Pathol 2011;28:273-77

23. Mrak RE, Yasargil MG, Mohapatra G, et al. Atypical extraventricular neurocytoma with oligodendroglioma-like spread and an unusual pattern of chromosome $\mathbf{1 p}$ and $\mathbf{1 9} \mathbf{q}$ loss. Hum Pathol 2004;35: 1156-59

24. Chang $\mathrm{KH}, \mathrm{Han} \mathrm{MH}$, Kim DG, et al. MR appearance of central neurocytoma. Acta Radiol 1993;34:520-26

25. Wichmann W, Schubiger O, von Deimling A, et al. Neuroradiology of central neurocytoma. Neuroradiology 1991;33:143-48

26. Kocaoglu M, Ors F, Bulakbasi N, et al. Central neurocytoma: proton MR spectroscopy and diffusion weighted MR imaging findings. Magn Reson Imaging 2009;27:434-40

27. Guo AC, Cummings TJ, Dash RC, et al. Lymphomas and high-grade astrocytomas: comparison of water diffusibility and histologic characteristics. Radiology 2002;224:177-83

28. Rumboldt Z, Camacho DL, Lake D, et al. Apparent diffusion coefficients for differentiation of cerebellar tumors in children. AJNR Am J Neuroradiol 2006;27:1362-69

29. Aralasmak A, Karaali K, Akyuz M, et al. MR imaging and CT perfusion findings of an extraventricular neurocytoma. Eur J Radiol Extra 2009;69:e53-e56

30. Koeller KK, Rushing EJ. From the archives of the AFIP: pilocytic astrocytoma: radiologic-pathologic correlation. Radiographics 2004;24:1693-708

31. Kumar AJ, Leeds NE, Kumar VA, et al. Magnetic resonance imaging features of pilocytic astrocytoma of the brain mimicking highgrade gliomas. J Comput Assist Tomogr 2010;34:601-11

32. Jayasundar $R$, Shah $T$, Vaishya $S$, et al. In vivo and in vitro MR spectroscopic profile of central neurocytomas. J Magn Reson Imaging 2003; $17: 256-60$ 